\title{
Joint meeting between the Royal College of Psychiatrists and the American Board of Psychiatry and Neurology (ABPN), 6 July 1996
}

\author{
Anne Dean
}

The following summarises discussions which took place at the above meeting, regarding provision of psychiatric care in the United States.

The method of accreditation (i.e. approving training) in the United States is totally independent of government intervention. Residency Review Committees (RRCs) have been established for all medical specialities and some subspecialities, and training schemes are visited at least every five years with a view to inspecting their standards and training programmes. These are closely monitored to ensure that such recommendations are implemented.

General training in psychiatry consists of one year of general medical training which includes some psychiatry and neurology. This is followed by a further three in general psychiatry or neurology, and includes various aspects of subspeciality training. The most developed subspeciality in the US is Child and Adolescent Psychiatry, which requires two years of training beginning either in the fourth or fifth year. For the new sub-specialities, which include Geriatric, Addiction and Forensic Psychiatry, the postresident training schemes are of one year's duration and lead to certification by the American Board. The American Board of Medical Specialities is responsible for the oversight of 24 medical Boards including the ABPN.

Medical care in the United States is provided in the following way, with financial considerations being emphasised to a far greater degree than in the UK.

Private - patient pays for all medical expenses:

Commercial insurance - various plans and benefit limits exist - co-payment patient:

Medicare - this is available to individuals 65 years and over/younger disabled persons;

Medicaid - available to poor women and children and very poor others:

Charity care - available to those too rich (or not registered) for Medicaid but still poor;

State - chronic/severe mentally ill.

\section{Psychiatric residency training}

This is funded in four ways; however, with managed care developing rapidly in the US, the future of residency training funding is more in doubt.

\section{Medicare funded}

State, County and other Charity patients

Third party (insurance) funded

Same role as always in private settings

\section{Psychiatric reimbursement by payors}

Treatment usually limited to 20-30 out-patient visits and 30 days' hospitalisation maximum. Usually non-discriminated treatment of alcohol and substance abuse $\mathbf{- 3 0}$ day programme basic.

\section{Training for primary care doctors}

There is a need for psychiatrists to have considerable involvement in the training of family doctors (GPs).

\section{Referral mechanisms for psychiatric care include the following}

Patients can refer themselves directly to psychiatrist.

Patients can approach primary care doctor (GP) who may approve referral to psychiatrist.

Primary care non-physicians can act as gatekeepers - i.e. social workers, etc.

Changes in the medical school curriculum in the US will hopefully lead to the recruitment of more primary care physicians, and there is an increased emphasis on the need for psychiatric input at an early stage.

Anne Dean, Head of Postgraduate Educational Services, Royal College of Psychiatrists, London 